\title{
Activity of the Akt-dependent anabolic and catabolic pathways in muscle and liver samples in cancer-related cachexia
}

Thomas L. Schmitt • Marcus E. Martignoni • Jeannine Bachmann • Kerstin Fechtner • Helmut Fries • Ralf Kinscherf • Wulf Hildebrandt

Published online: 22 May 2007

(C) Springer-Verlag 2007

Erratum to: J Mol Med (2007)

DOI 10.1007/s00109-007-0177-2

Unfortunately, Figs. 2, 3, 4 and 5 were printed with errors. The corrected figures are given here:

The online version of the original article can be found at http://dx.doi. org/10.1007/s00109-007-0177-2.

T. L. Schmitt $(\bowtie) \cdot$ W. Hildebrandt

Division of Immunochemistry (D020),

German Cancer Research Center,

Im Neuenheimer Feld 280,

69120 Heidelberg, Germany

e-mail: tlrp.schmitt@web.de

M. E. Martignoni $\cdot$ J. Bachmann $\cdot$ H. Fries

Department of General Surgery, University of Heidelberg,

Heidelberg, Germany

K. Fechtner

Department of Radiology, University of Heidelberg,

Heidelberg, Germany

R. Kinscherf

Anatomy and Developmental Biology, Medizinische Fakultät

Mannheim, University of Heidelberg,

Heidelberg, Germany 

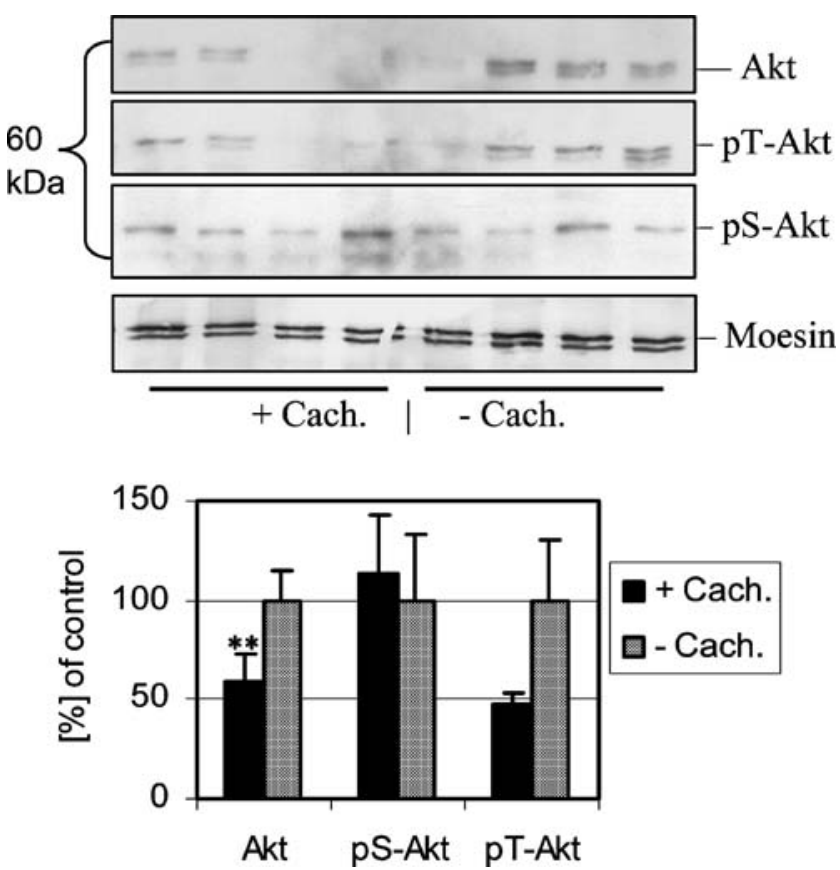

Fig. 2 Western blot analysis of Akt expression and phosphorylation in muscle biopsies of cachectic (+Cach.) and non-cachectic (-Cach.) patients. The blot shows representative lysates that were separated on a $9 \%$ SDS-PAA gel and stained with antibodies directed against all isoforms of $\mathrm{Akt}, \mathrm{pS}^{473}$-Akt or $\mathrm{pT}^{308}$-Akt. In the lower panel, the percentage of relative protein level is reported as mean \pm SEM, where the value from non-cachectic subjects (control group) is set to $100 \%$. $* * P \leq 0.01$
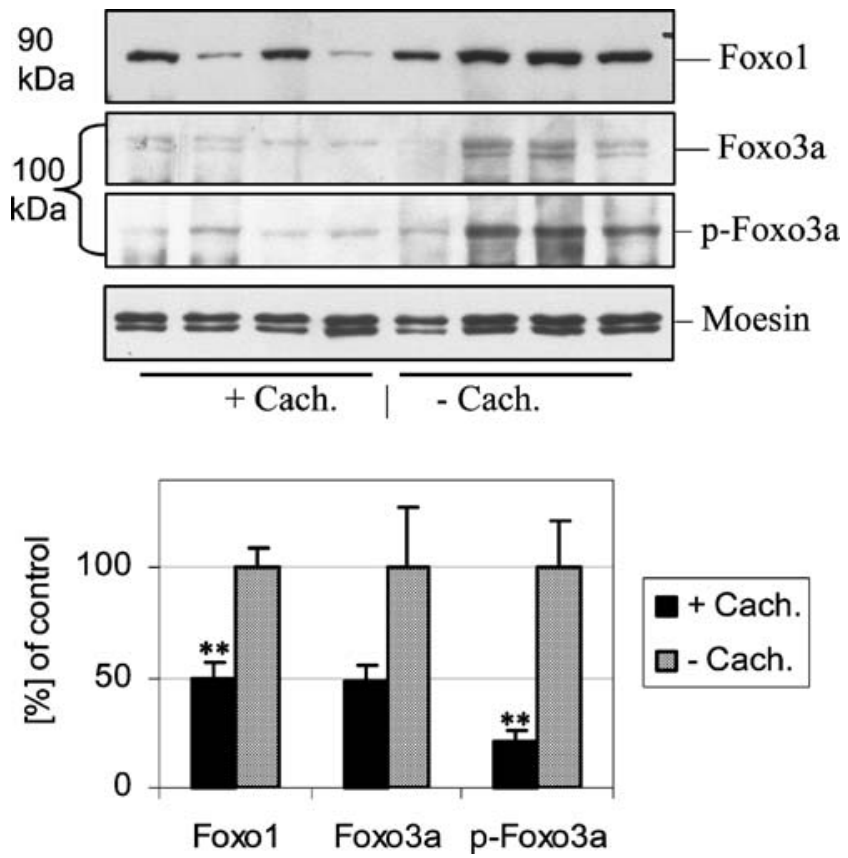

Fig. 3 Western blot analysis of expression and phosphorylation of Foxo proteins in muscle biopsies of cachectic $(+\mathrm{Cach}$.) and noncachectic (-Cach.) patients. The blot shows representative lysates that were separated on a $9 \%$ SDS-PAA gel and stained with antibodies directed against Foxo1 (FKHR), Foxo3a (FKHRL1) and $\mathrm{pThr}^{32}$ Foxo3a, respectively. In the lower panel, the percentage of relative protein level is reported as mean $\pm \mathrm{SEM}$, where the value from noncachectic subjects (control group) is set to $100 \%$. ${ }^{* *} P \leq 0.01$
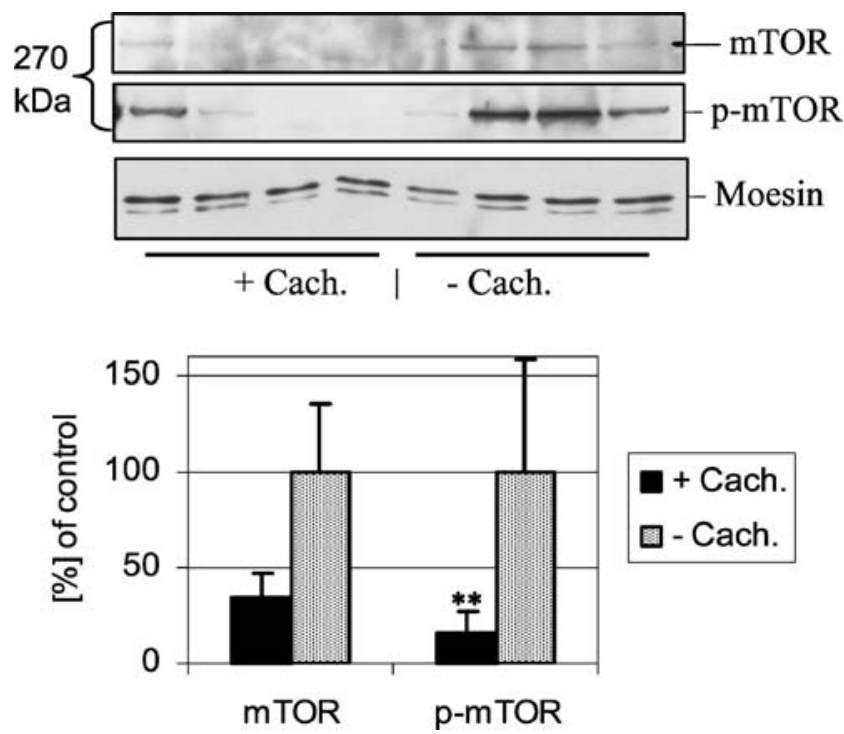

Fig. 4 Western blot analysis of mTOR expression and phosphorylation in muscle biopsies of cachectic (+Cach.) and non-cachectic (-Cach.) patients. Representative lysates were separated on a $7.5 \%$ SDS-PAA gel and stained with antibodies directed against mTOR and $\mathrm{pS}^{2448}$-mTOR. In the lower panel, the percentage of relative protein level is reported as mean $\pm \mathrm{SEM}$, where the value from non-cachectic subjects as the control group is set to $100 \%$. ${ }^{* *} P \leq 0.01$
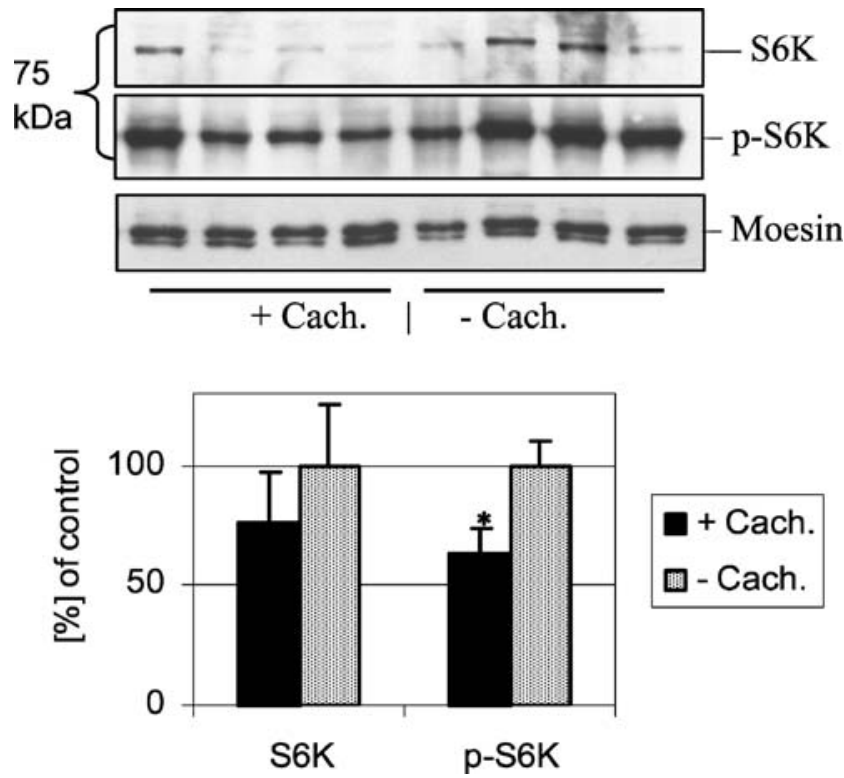

Fig. 5 Western blot analysis of expression and phosphorylation of p70S6 kinase in muscle biopsies of cachectic (+Cach.) and noncachectic $(-$ Cach. $)$ patients. The blot shows representative muscle lysates from muscle that were separated on a 9\% PAA gel and probed with antibodies directed against $\mathrm{S} 6 \mathrm{~K}$ and $\mathrm{pT}^{389}-\mathrm{S} 6 \mathrm{~K}$. In the lower panel, the percentage of relative protein level is reported as mean \pm SEM, where the value from non-cachectic subjects (control group) is set to $100 \%$. $* P \leq 0.05$ 